\title{
Comportamento reológico de iogurte de cajá comercializado em Campina Grande, Paraíba
}

\section{Rheological properties of yogurt cajá marketed in Campina Grande, state Paraíba}

\author{
Flávio Farias Gurjão ${ }^{1}$, Gilmara Gurjão Carneiro ${ }^{2}$, Taciano Pessoa ${ }^{3}$, Débora Rafaelly Soares Silva ${ }^{4}$, Patricia Rodrigues Pêe ${ }^{5}$
}

Resumo: O estudo reológico contribui para o conhecimento da estrutura molecular, no controle de qualidade e aceitação de um determinado produto, auxilia no controle do processo industrial e em projetos de equipamentos no processamento dos materiais. Objetivou-se avaliar o comportamento reológico de iogurte de cajá e a adequação dos dados reológicos aos modelos de Ostwald-de-Waele, Herschel-Bulkley e Mizrahi-Berk e ainda o efeito da temperatura sobre o comportamento reológico. Os dados reológicos foram obtidos através de um viscosímetro Brookfield DV-II+Pro. Os ensaios foram realizados nas temperaturas 20, 30 e $40{ }^{\circ} \mathrm{C}$ e os resultados experimentais foram ajustados pelos modelos de Ostwalde-de-Waelle, Casson, Hershel Bulkey e Mizrahi-Berk, com auxílio do software STATISTICA, versão 7.0. O iogurte analisado neste experimento apresentou comportamento de fluido não newtoniano com características pseudoplástica. Os modelos reológicos de Hershel Bulkey e Mizrahi-Berk representaram satisfatoriamente o comportamento reológico do iogurte de cajá nas diferentes temperaturas estudadas, apresentando coeficientes de correlação $\left(\mathrm{R}^{2}\right)$, acima de 0,99 .

Palavras-chave: qualidade, reologia, temperatura.

\begin{abstract}
The rheological study contributes to the knowledge of the molecular structure, the quality control and acceptance of a particular product, helps to control the manufacturing process and equipment designs in the processing of materials. This study aimed to evaluate the rheological behavior of yogurt cajá and the adequacy of the rheological data to models of Ostwaldde-Waele, Herschel-Bulkley and Mizrahi-Berk still the effect of temperature on the rheological behavior. The rheological data were obtained using a Brookfield DV-II + Pro. Assays were carried out at temperatures 20,30 and $40{ }^{\circ} \mathrm{C}$ and the experimental results were adjusted by the model-to-Ostwalde Waelle, Casson and Herschel Bulkey Mizrahi-Berk, using the STATISTICA software, version 7.0. The yogurt analyzed in this experiment showed behavior of pseudoplastic non-Newtonian fluid characteristics. The rheological models Hershel Bulkey and Mizrahi-Berk satisfactorily represented the rheological behavior of the hog plum yogurt at different temperatures studied, with correlation coefficients $\left(\mathrm{R}^{2}\right)$ above 0.99 .
\end{abstract}

Keywords: quality, rheology, temperature.

\footnotetext{
*Autor para correspondência

Recebido para publicação em 23/02/2015; aprovado em 30/06/2015

${ }^{1}$ Doutorando em Engenharia de Processos, UFCG, Campina Grande, PB. Fone: (83) 3333-3620, flavioggurjao@hotmail.com;

${ }^{2}$ Doutoranda em Engenharia de Processos, UFCG, Campina Grande, PB. Fone: (83) 99226380, gilmaragurjao@ hotmail.com;

${ }^{3}$ Doutorando em Engenharia de Processos, UFCG, Campina Grande, PB. Fone: (83) 8825-9332, pessoat@hotmail.com;

${ }^{4}$ Doutoranda em Engenharia de Processos, UFCG, Campina Grande, PB. Fone: (83), deborarafaelly@yahoo.com.br;

${ }^{5}$ Dra. em Engenharia Agrícola, Professora da Escola Técnica Redentorista, ETER, Campina Grande, PB. Fone: (83) 3333.1331 patriciarodriguespe@yahoo.com.br.
} 


\section{INTRODUÇÃO}

O iogurte é um produto lácteo fresco, obtido pela fermentação do leite com cultivos pró-simbióticos das bactérias Streptococcus termophilus e Lactobacillus bulgaricus. Surgiu no Oriente e depois entre os gregos e romanos. Esse alimento, que hoje faz parte do cotidiano da maioria das pessoas, rapidamente se difundiu, conquistando uma posição privilegiada nas dietas alimentares dos mais diversos povos (CIRIBELI; CASTRO, 2011).

A reologia é definida como a ciência que estuda como a matéria se deforma ou escoa, quando submetida a esforços produzidos por forças externas (MACHADO, 1996).

O comportamento reológico representa o comportamento mecânico dos materiais quando em processo de deformação devido a um campo de tensões. A reologia tem importância nos fenômenos relacionados à transferência de massa que tem lugar nos processos industriais, durante o processo de elaboração como concentração, mistura pasteurização e outras operações; as características reológicas também são imprescindíveis na otimização, no controle e nos cálculos de processos. Esses conhecimentos servem, igualmente, para o desenvolvimento de produtos alimentícios e correlação de parâmetros físicos e sensoriais A variabilidade do comportamento reológico está relacionada com a alteração estrutural provocada pelo cisalhamento (FERREIRA et al., 2002).

Um fluido define-se como uma substância que se deforma continuamente quando se submete a um esforço constante, sem se importar quão pequeno seja este esforço (HAMINIUK, 2005).

Viscosidade é a propriedade física de um líquido de resistir ao fluxo induzido pelo cisalhamento; é dependente da natureza físico-química da substância, da temperatura da substância, da pressão, da taxa de cisalhamento e do tempo (SCHARAMM, 2000).

O comportamento reológico dos fluidos viscosos está divido em Newtonianos e não Newtonianos, dependendo da relação entre tensão cisalhante e a taxa de cisalhamento (MACHADO, 1996).

Objetivou-se avaliar o comportamento reológico de iogurte de cajá e a adequação dos dados reológicos aos modelos de Ostwald-de-Waele, Herschel-Bulkley e MizrahiBerk e ainda o efeito da temperatura sobre o comportamento reológico.

\section{MATERIAL E METODOS}

O experimental foi conduzido no Laboratório de Armazenamento e Processamento de Produtos Agrícolas (LAPPA) da Unidade Acadêmica de Engenharia Agrícola, da Universidade Federal de Campina Grande - PB.

Utilizou-se no experimento iogurte de cajá (Spondias spp.), adquiridos no comércio local de Campina Grande-PB.

As medidas reológicas foram realizadas utilizando-se um viscosímetro Brookfield modelo DV- II + Pro, fabricado por Brookfield Engineering Laboratories, E.U.A nas temperaturas 20,30 e $40^{\circ} \mathrm{C}$, em triplicata, utilizando-se um spindle número 2, nas velocidades de rotação 5,0, 10, 20, 30, $40,50,60,70,80,90,100,120,140,160 \mathrm{rpm}$. Para a realização do experimento, foi utilizado um béquer de 600 $\mathrm{mL}$ onde foram colocadas $600 \mathrm{~mL}$ de amostra para obtenção das leituras no viscosímetro.
Os dados experimentais obtidos no viscosímetro para as diferentes temperaturas foram calculadas as tensões de cisalhamento e as taxas de deformação, para as diferentes velocidades de rotação e para as diferentes temperaturas, utilizando-se a metodologia proposta por Mitschka (1982), os quais foram correlacionados aos os modelos reológicos de Ostwald-de-Waelle ou Lei da potência, Casson, HerschelBulkley, e Mizrahi-Berk, utilizando-se o programa computacional Statistica versão 7.0. Para a determinação do melhor ajuste analisou-se o coeficiente de determinação $\left(\mathrm{R}^{2}\right)$, e o desvio percentual médio $(\mathrm{P})$.

\section{RESULTADOS E DISCUSÃO}

Na Tabela 1 são apresentados os parâmetros dos modelos reológicos de Ostwald-de-Waele, (Lei da potência), Casson, Herschel-Bulkley e Mizrahi-Berk, ajustados aos dados experimentais de tensão de cisalhamento versus taxa de deformação do iogurte de Polpa de cajá nas temperaturas de 20,30 e $40^{\circ} \mathrm{C}$, bem como os coeficientes de determinação $\left(\mathrm{R}^{2}\right)$. Verifica-se que todos os modelos aplicados indicaram coeficientes de determinação $\left(\mathbf{R}^{2}\right)$ acima de 0,97 , resultando em bom ajuste dos dados de tensão de cisalhamento e taxa de deformação do iogurte de caja, nas três temperaturas analisadas.

Os modelos de Herschel-Bulkley e MizrahiBerk.apresentaram os melhores ajustes, com os maiores coeficientes de determinação $(\mathrm{R})^{2}$ superiores a 0,99 e os menores desvios percentuais médios, inferiores a $1,39 \%$.

Verifica-se que, no modelo de Ostwald-de-Waele, o índice de consistência (K), diminuiu com o aumento da temperatura; no entanto, observa-se também que houve um aumento pontual, na temperatura de $40^{\circ} \mathrm{C}$. No modelo de Casson o parâmetro $\mathbf{K}_{\mathbf{O C}}$ (tensão de cisalhamento inicial) diminuiu com o aumento da temperatura, tendência também observada por Oliveira et al. (2008) ao avaliar o comportamento reológico de amostras de sorvetes de iogurte, cujos valores variaram de 0,2927 a 0,2172, nas temperaturas de -2 a $2{ }^{\circ} \mathrm{C}$; No entanto, observa-se um aumento da tensão de cisalhamento na temperatura a $40^{\circ} \mathrm{C}$.

Os valores da viscosidade plástica de Casson $\mathbf{K}_{\mathbf{C}}$ demonstraram tendência de diminuição com o aumento da temperatura. Os índices de consistência, $\mathbf{K}_{\mathbf{H}}$ determinados a partir dos modelos de Herschel-Bulkley, também mostraram tendência de decrescer com a elevação da temperatura. Haminiuk (2005) encontrou comportamento semelhante para o parâmetro K ao ajustar o modelo de Herschel-Bulkley aos dados experimentais da polpa de amora-preta integral para a faixa de temperatura entre 10 e $60{ }^{\circ} \mathrm{C}$; Oliveira (2006) encontrou comportamento oposto para polpa de pitanga integral nas temperaturas de 10 a $50{ }^{\circ} \mathrm{C}$, onde ocorreu um aumento do índice de consistência com a elevação da temperatura.

$\mathrm{O}$ índice de consistência $\mathbf{K}_{\mathbf{M}}$, determinado a partir do modelo de Mizrahi-Berk, também mostrou tendência de decrescer com a elevação da temperatura, no entanto observase que houve um aumento pontual, na temperatura de $40^{\circ} \mathrm{C}$. A tensão de cisalhamento inicial $\mathbf{K}_{\mathbf{0 M}}$ (raiz quadrada da tensão inicial) no modelo de Mizrahi-Berk apresentou tendência de diminuição com o aumento da temperatura; comportamento semelhante foi encontrado por Oliveira (2006) estudando polpa de pitanga integral. 
Tabela 1- Parâmetros de ajuste dos modelos reológicos ajustados aos dados experimentais da tensão de cisalhamento, em função da taxa de deformação de iogurte de polpa de cajá

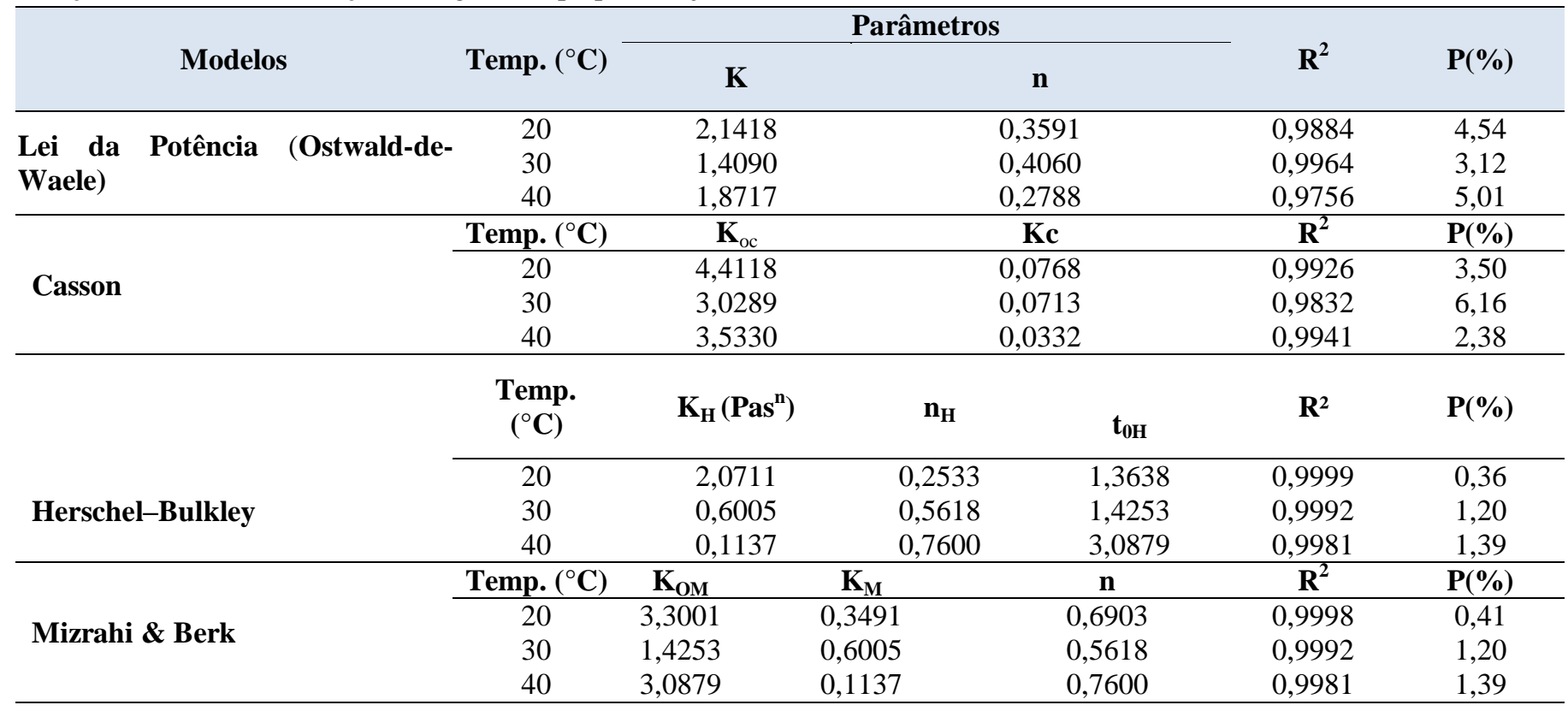

Para o parâmetro $\mathbf{n}$ (índice de comportamento do fluido) dos modelos de Ostwald-de-Waele, (Lei da potência), Casson, Herschel-Bulkley e Mizrahi-Berk, verifica-se que os valores ficaram abaixo do valor da unidade, o que caracteriza as amostras como fluidos não newtonianos com características pseudoplásticas, sabendo-se que quanto mais distante o $\mathbf{n}$ estiver do valor unitário, mais distante o fluido também estará do comportamento newtoniano. $\mathrm{Na}$ indústria este comportamento se reflete no aumento de velocidade de fluxo quando submetido a estreitamento de tubos (por exemplo, envase) e possibilidade de intensa homogeneização em processos de mistura (BEZERRA, 2010). Fluidos nãonewtonianos apresentando valores de $\mathbf{n}$ abaixo de um, foram observados por Cabral et al. (2002) para polpa de cupuaçu; por Carneiro (2011) ao avaliar o comportamento reológico de geleias de pimenta; por Bezerra (2010) ao avaliar o comportamento reológico de iogurtes de leite de cabra, de búfala e de sua mistura.

Nas Figuras 1 a 4 são apresentadas as curvas de ajuste dos dados experimentais de tensão de cisalhamento em função da taxa de deformação, ajustadas pelos modelos Ostwald-de-Waele (Lei da potência), Casson, HerschelBulkley e Mizrahi-Berk nas temperaturas de 20, 30 e $40^{\circ} \mathrm{C}$ para o iogurte de cajá. Observa-se que para uma dada tensão de cisalhamento a taxa de deformação a diminui com o aumento da temperatura. Bezerra (2010) ao avaliar o comportamento reológico de iogurtes na temperatura de $5^{\circ} \mathrm{C}$, observou um comportamento semelhante. Verifica-se que as curvas não apresentaram uma relação linear entre a tensão de cisalhamento e a taxa de deformação, caracterizando o iogurte de cajá como fluidos não newtonianos.
Figura 1- Relação entre tensão de cisalhamento e taxa de deformação para o iogurte de cajá, nas temperaturas 20, 30 e $40^{\circ} \mathrm{C}$, descrita pelo modelo de Ostwald-de-Waelle

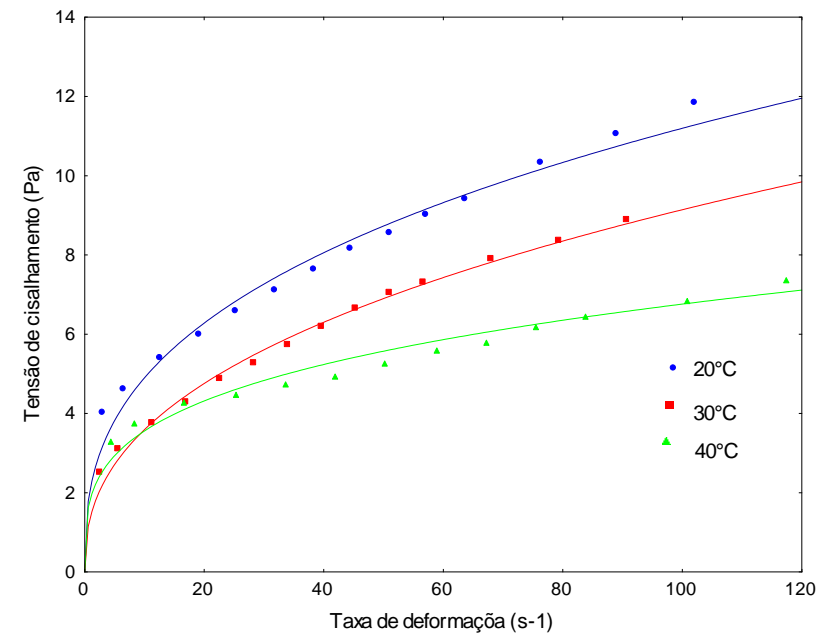

Figura 2- Relação entre tensão de cisalhamento e taxa de deformação para o iogurte de cajá, nas temperaturas 20, 30 e $40^{\circ} \mathrm{C}$, descrita pelo modelo de Casson

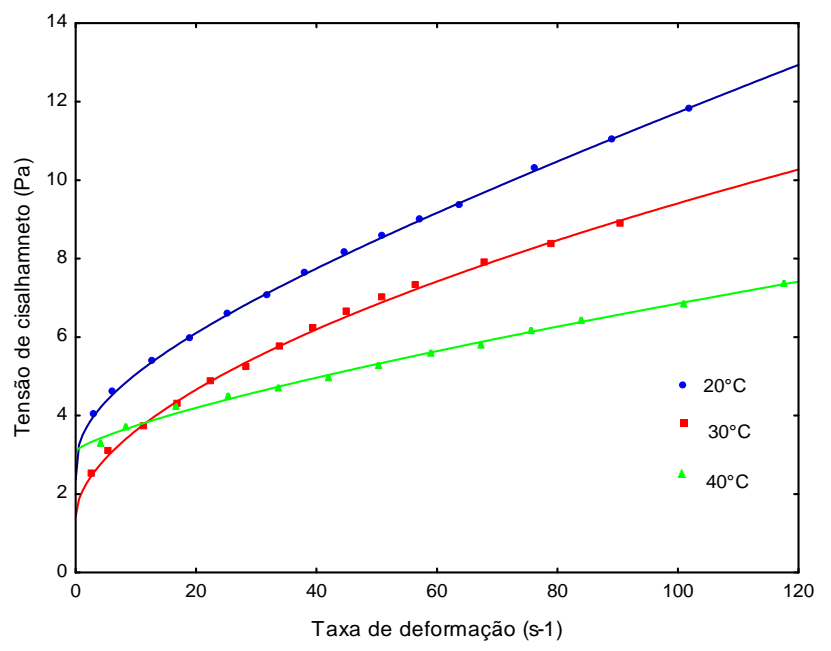


Figura 3- Relação entre tensão de cisalhamento e taxa de deformação para o iogurte de cajá, nas temperaturas 20, 30 e $40^{\circ} \mathrm{C}$, descrita pelo modelo de Herschel-Bulkley

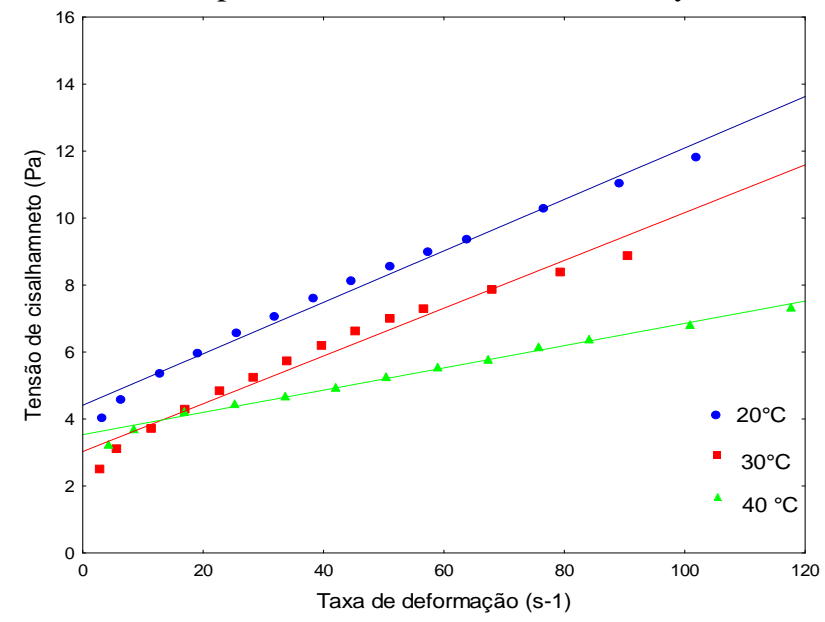

Figura 4- Relação entre tensão de cisalhamento e taxa de deformação para o iogurte de cajá, nas temperaturas 20, 30 e $40^{\circ} \mathrm{C}$, descrita pelo modelo de Mizrahi \& Berk

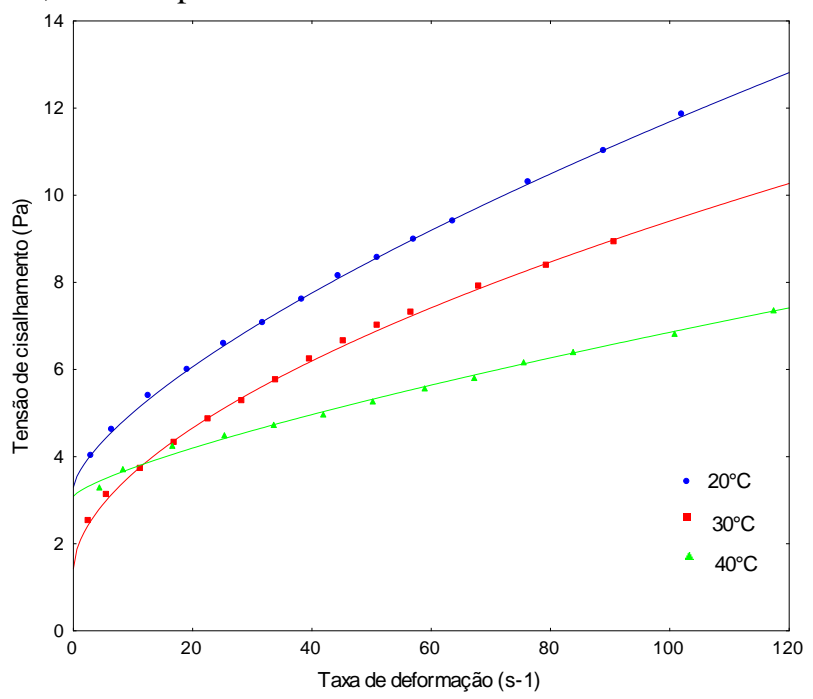

\section{CONCLUSÃO}

O iogurte de cajá apresentou comportamento de fluido não newtoniano com características pseudoplástica;

Os modelos reológicos Herschel-Bulkley e Mizrahi \& Berk apresentaram os melhores ajustes, com coeficiente de determinação $\left(\mathrm{R}^{2}\right)$, acima de 0,99 e os desvios percentuais médios, inferiores a $1,39 \%$.

\section{REFERÊNCIAS}

BEZERRA, M. F. Caracterização física química, reológica e sensorial de iogurte obtido pela mistura de leites bubalinos e caprinos. Natal: Universidade Federal do Rio Grande do Norte, 2010. 100 p. Dissertação de Mestrado

BRANDÃO, S. C.C.; Tecnologia da produção industrial de iogurte. XXXV Semana do laticinista - Instituto Cândido Tostes Juiz de Fora, 1997. 52 p.
CABRAL, M. F. P.; QUEIROZ, A. J. de M.; FIGUEIRÊDO, R. M. F. de. Comportamento reológico da polpa de cupuaçu (Theobroma grandiflorum schum.) peneirada. Revista Brasileira de Produtos Agroindustriais. Campina Grande, v. 4, n. 1, p. 37-40, 2002.

CARNEIRO, G. G. Elaboração de geléia de pimenta da variedade cambuci em diferentes estádios de maturação e concentração de sacarose. Campina Grande: Universidade Federal de Campina Grande. 2011. 128 p. Dissertação Mestrado

CIRIBELI, J. P.; CASTRO, L. S. de. Descrição da cadeia produtiva do iogurte: um estudo de caso realizado no Laticínio do Centro Federal de Educação Tecnológica de Rio Pomba. Revista Gestão Empresarial, Rio Pomba, v. 1, n. 1, p.75-87, 2011.

FERREIRA, G. M.; QUEIROZ, A. J. M.; CONCEIÇÃO, R. S.; GASPARETTO, C. A. Efeito da temperatura no comportamento reológico das polpas de caju e goiaba. Revista Ciências Exatas e Naturais, v. 4, n. 2, p. 175-184, 2002.

HAMINIUK, C. W. I. Comportamento reológico e fracionamento péctico das polpas integrais de araçá (psidium catlleianum sabine) e amora-preta (Rubus spp). Paraná: Universidade Federal do Paraná. 2005. 84. p. Dissertação Mestrado

MACHADO, J. C. V. Reologia, viscosidade e escoamento. Rio de Janeiro: Petrobrás/SEREC/CENNOR, 1996. 89p.

OLIVEIRA, F. M. N. DE. Secagem e armazenamento da polpa de pitanga. Campina Grande: Universidade Federal de Campina Grande. 2006. 197 p. Dissertação Mestrado

SCHARAMM, G. A pratical approach to rheology and rheometry. Germany: Karlsruhe, 2000. 291 p. 